\title{
Two-step demodulation based on the Gram-Schmidt orthonormalization method
}

\author{
J. Vargas,$^{1, *}$ J. Antonio Quiroga, ${ }^{2}$ C. O. S. Sorzano, ${ }^{1}$ J. C. Estrada, ${ }^{3}$ and J. M. Carazo ${ }^{1}$ \\ ${ }^{1}$ Biocomputing Unit, Centro Nacional de Biotecnología-CSIC, C/Darwin 3, 28049, Cantoblanco (Madrid), Spain \\ ${ }^{2}$ Optics Department, Universidad Complutense de Madrid, Facultad de CC. Físicas, Ciudad Universitaria s/n, 28040 Madrid, Spain \\ ${ }^{3}$ Centro de Investigaciones en Óptica A. C., Loma del Bosque 115, Col. Lomas del Campestre, 37150, León (Guanajuato), Mexico \\ *Corresponding author: jvargas@cnb.csic.es
}

Received October 5, 2011; revised December 13, 2011; accepted December 14, 2011;

posted December 14, 2011 (Doc. ID 156019); published February 1, 2012

\begin{abstract}
This Letter presents an efficient, fast, and straightforward two-step demodulating method based on a Gram-Schmidt (GS) orthonormalization approach. The phase-shift value has not to be known and can take any value inside the range $(0,2 \pi)$, excluding the singular case, where it corresponds to $\pi$. The proposed method is based on determining an orthonormalized interferogram basis from the two supplied interferograms using the GS method. We have applied the proposed method to simulated and experimental interferograms, obtaining satisfactory results. A complete MATLAB software package is provided at http://goo.gl/IZKF3. @ 2012 Optical Society of America OCIS codes: $\quad 100.5070,100.2650$.
\end{abstract}

In the past there have been reported works about phase reconstruction with only two frames [1-3]. In [1] is presented the standard and most used technique for obtaining the modulating phase map from two phase-shifted interferograms. The method is based on the application of the Fourier transform demodulating approach to both interferograms. Then, the phase-step map is calculated using a direct algebraic expression. As the phase step has to be equal for all pixels, it is possible to solve the local sign ambiguity and, therefore, retrieve the phase map. This method requires filtering out the DC term, but it does not need the normalization of the fringe patterns. The main drawback of this approach is that it is very sensitive to noise. In [2] is presented a self-tuning (SF) method that first retrieves the phase step between interferograms, looking for the minimum of a merit function. Then a quadrature filter is constructed from the obtained phase step and the modulating phase is determined. This method presents good results when the phase step is close to $\pi / 2 \mathrm{rad}$, but the accuracy decreases when the phase step moves away from this value. Additionally, the method requires the interferograms to be previously normalized. In [3] is presented a recent demodulating two-step method based on a regularized optical-flow (OF) method. The method is robust against additive noise and different values of the phase step. Additionally, this approach does not require normalizing the fringe patterns but it requires subtracting the DC term. The main drawback of [3] resides on the computational requirements necessary to perform the $\mathrm{OF}$ analysis, which make this demodulating method costly from a processing and computational point of view.

In this work, we present a novel two-step demodulation method based on the Gram-Schmidt (GS) orthonormalization approach. The method is very fast, easy to implement, does not require any minimization process, and is not computationally demanding. The method requires filtering out the DC term, but it does not require normalizing the fringe patterns. In [4-5] we have shown that a sequence of phase-shifted fringe patterns free from harmonics can be expressed as a linear combination of two orthonormal signals. Therefore, any phase-shifted interferogram sequence can be described using a twodimensional vector subspace. The orthonormal signals form a basis of the interferograms sequence. If the interferograms have more than one fringe, these orthonormal signals are also in quadrature. Therefore, there is a deep relation between demodulation and orthonormalization processes. In this Letter we have used this idea to propose a novel two-step demodulation approach based on the GS orthonormalization method.

In phase-shifting interferometry, an interferogram sequence can be described by the following expression:

$$
I_{n}(x, y)=a(x, y)+b(x, y) \cos \left[\Phi(x, y)+\delta_{n}\right]
$$

where $a(x, y)$ is the DC component, $b(x, y)$ and $\Phi(x, y)$ are the modulation and phase maps, and $\delta_{n}$ are the phase steps. In our case of interest, $n \in[1,2]$, Eq. (1) can be rewritten as

$$
I_{n}=a+b\left(\cos [\Phi] \cos \left[\delta_{n}\right]-\sin [\Phi] \sin \left[\delta_{n}\right]\right)
$$

From Eq. (2) and grouping terms we obtain

$$
I_{n}=a+\alpha_{n} I_{c}+\beta_{n} I_{s},
$$

where $\alpha_{n}=\cos \left[\delta_{n}\right], \quad \beta_{n}=\sin \left[\delta_{n}\right], \quad I_{c}=b \cos [\Phi], \quad I_{s}=$ $-b \sin [\Phi]$, and the spatial dependence has been omitted for the sake of clarity. In Eq. (3), we show that any interferogram can be decomposed into three signals. Typically, the DC signal is a smooth signal that can be filtered out. Therefore, any DC filtered interferogram can be decomposed into two signals.

The GS process is a method for orthonormalizing a set of vectors [6]. This approach takes a finite vector set, $S=\left\{u_{1}, \ldots, u_{j}\right\}$, and generates from it an orthonormal set $\tilde{S}=\left\{\tilde{u}_{1}, \ldots, \tilde{u}_{k}\right\}$, with $k \leq j$, where $j$ and $k$ are the number of vectors that compose the $S$ and $\tilde{S}$ sets, respectively. In the case of orthonormalizing two vectors, the process is simple and consists of three steps. First, we take one of the two vectors and we normalize it: 


$$
\tilde{u}_{1}=u_{1} / \sqrt{\left\langle u_{1}, u_{1}\right\rangle}=u_{1} /\left\|u_{1}\right\| .
$$

Then, we orthogonalize $u_{2}$ with respect to the $\tilde{u}_{1}$ vector, subtracting its projection as

$$
\hat{u}_{2}=u_{2}-\left\langle u_{2}, \tilde{u}_{1}\right\rangle \cdot \tilde{u}_{1} .
$$

Finally, we obtain $\tilde{u}_{2}$ by dividing $\hat{u}_{2}$ by its norm as

$$
\tilde{u}_{2}=\hat{u}_{2} / \sqrt{\left\langle\hat{u}_{2}, \hat{u}_{2}\right\rangle}=\hat{u}_{2} /\left\|\hat{u}_{2}\right\| \text {. }
$$

Note that, in Eqs. (4), (5), and (6), the operator $\langle\cdot, \cdot\rangle$ is determined by the defined inner product. We define the inner product as

$$
\left\langle I_{1}(x, y), I_{2}(x, y)\right\rangle=\sum_{x=1}^{N_{x}} \sum_{y=1}^{N_{y}} I_{1}(x, y) I_{2}(x, y),
$$

where $N_{x}$ and $N_{y}$ corresponds to the image columns and rows, respectively. From Eqs. (4)-(7) we can obtain two orthonormal signals, $\tilde{I}_{1}$ and $\tilde{I}_{2}$, from the DC-filtered interferograms $I_{1}$ and $I_{2}$. Following the GS method outlined below, we have that

$$
\tilde{I}_{1}=b \cos (\Phi) / \kappa_{1}=b \cos (\Phi) / \sqrt{\sum_{x=1}^{N_{x}} \sum_{y=1}^{N_{y}}(b \cos (\Phi))^{2}} .
$$

We have considered that $\delta_{1}=0$ and $\delta_{2}=\delta=\left(\delta_{2}-\delta_{1}\right)$ without loss of generality because this phase-step origin will introduce an irrelevant piston term in the recovered phase. Additionally, following Eq. (5) (second step of the GS method), we can obtain $\hat{I}_{2}$, the nonnormalized version of $\tilde{I}_{2}$, as

$$
\begin{aligned}
\hat{I}_{2}= & b \cos (\Phi+\delta) \\
& -\left(\sum_{x=1}^{N_{x}} \sum_{y=1}^{N_{y}} b^{2} \cos (\Phi+\delta) \cos (\Phi)\right) b \cos (\Phi) / \kappa_{1}^{2} .
\end{aligned}
$$

If we have more than one fringe in the interferograms, we can use the approximation $\left|\sum_{x=1}^{N_{x}} \sum_{y=1}^{N_{y}} \cos ^{2}(\Phi) \cos (\delta)\right| \gg$ $\left|\sum_{x=1}^{N_{x}} \sum_{y=1}^{N_{y}} \cos (\Phi) \sin (\Phi) \sin (\delta)\right|$, and, therefore, we can rewrite Eq. (9) as

$$
\hat{I}_{2} \cong-b \sin (\Phi) \sin (\delta) .
$$

Finally, we obtain $\tilde{I}_{2}$ by dividing $\hat{I}_{2}$ by its norm. From Eq. (6) we have

$$
\tilde{I}_{2}=-b \sin (\Phi) / \kappa_{2}=-b \sin (\Phi) / \sqrt{\sum_{x=1}^{N_{x}} \sum_{y=1}^{N_{y}}(b \sin (\Phi))^{2}} .
$$

Note that $\hat{I}_{2}$ in Eq. (10) will have low values if $\delta$ is close to zero or $\pi$. In these cases, this signal will be highly affected by the noise. Additionally, we do not consider the case where the interferograms are affected by harmonics. In this case, Eqs. (1) and (3) are not valid. If there is more than one fringe in the interferograms, we have $\kappa_{1} \cong \kappa_{2}$ and the $\left(\tilde{I}_{1}, \tilde{I}_{2}\right)$ signals are in quadrature. Therefore, the modulating phase can be retrieved directly by an arctangent function as

$$
\Phi \cong \arctan \left(-\tilde{I}_{1} / \tilde{I}_{2}\right)
$$

To show the performance of the proposed method, we have tested it with simulated fringe patterns. We have compared the results obtained by the proposed GS method with the results obtained by the standard Kreis [1] , ST [2] , and OF []ㅡ methods. The utility of this method has special interest in the case of closed-fringe interferograms, as two frames are the minimum number of interferograms to reconstruct the phase without local sign ambiguity. In Fig. 1 we show two sample fringe patterns with a phase shift between them of $1 \mathrm{rad}$.

The noise is Gaussian and additive, and the signal-tonoise ratio is of $4 \%$. In Fig. 2 is shown the reference theoretical phase map and in Fig. 3 are shown the phases obtained by (a) the proposed GS method and the (b) ST, (c) Kreis, and (d) OF methods. Observe in Fig. 3 that the wrapped phase retrieved by the different methods is very similar. In Table 1 we show the different rootmean-square errors (rms) of the difference between the theoretical and obtained wrapped phases and processing times. The processing times have been obtained using a $2.67 \mathrm{GHz}$ laptop and processing with MATLAB.

We have applied the proposed algorithm to real interferograms. We have compared the retrieved phase from two consecutive frames using the proposed GS method and the standard Kreis, the ST, and the OF methods with the phase retrieved by a set of 19 interferograms, using the advanced iterative algorithm (AIA) method [7]. In Fig. $\underline{4}$ we show the two interferograms used by the two-step methods.
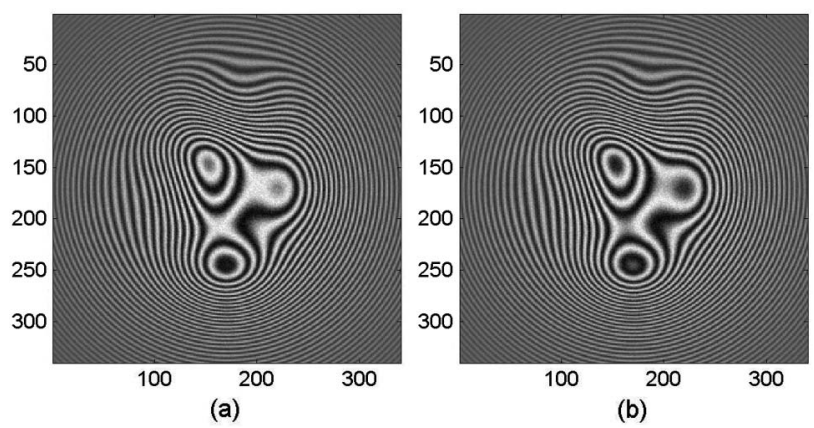

Fig. 1. Two fringe patterns used in the first simulation.

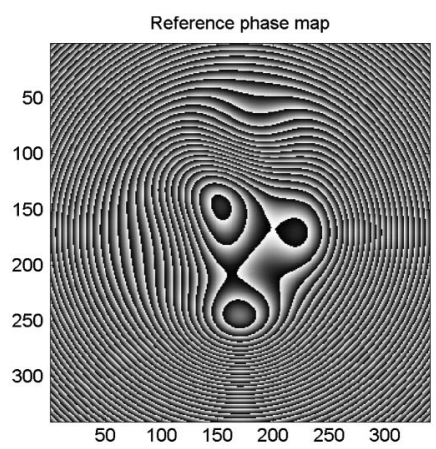

Fig. 2. Theoretical phase map of the simulated fringe patterns. 

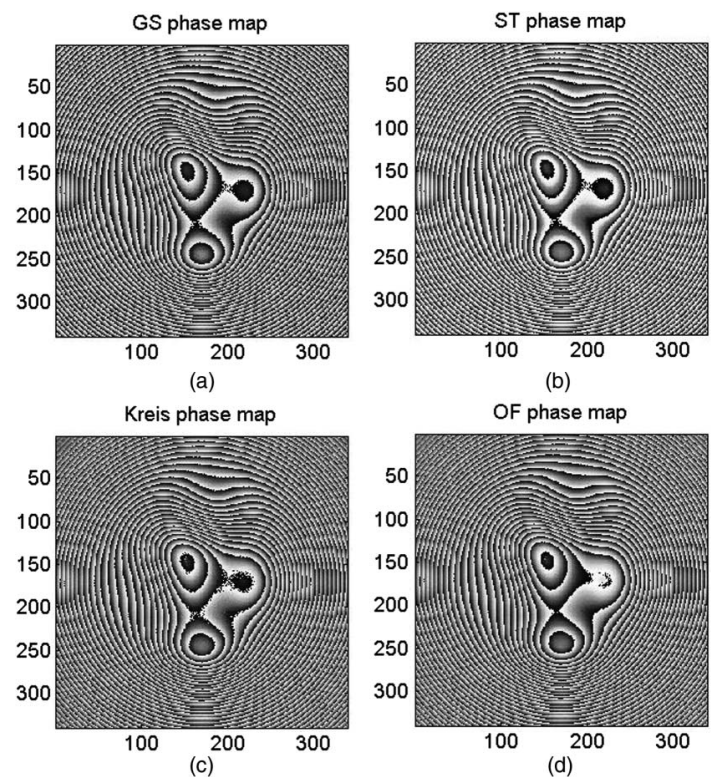

Fig. 3. Reconstructed wrapped phases by (a) the proposed GS method and the (b) ST, (c) Kreis, and (d) OF methods.

Table 1. Root-Mean-Square Errors and Processing Times Obtained by the GS, Kreis, ST, and OF Methods in the Simulation

\begin{tabular}{llccc}
\hline & GS & Kreis & ST & OF \\
\hline rms (rad) & 0.21 & 0.25 & 0.32 & 0.25 \\
Time (s) & 0.025 & 0.20 & 0.92 & 0.52 \\
\hline
\end{tabular}
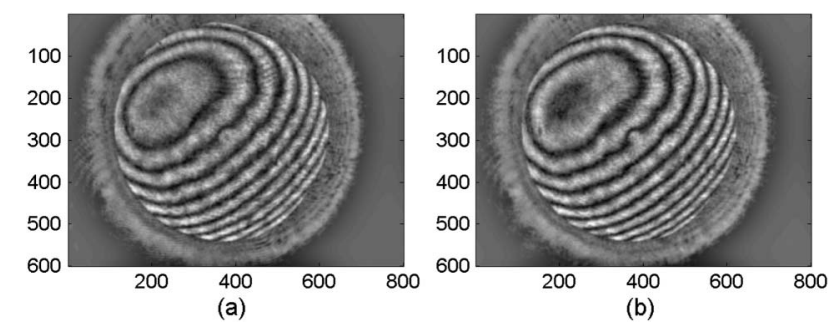

Fig. 4. Two sample real interferograms used.

In Fig. 5 we show the reference phase obtained by the AIA algorithm and 19 interferograms. Figure 6 shows the obtained phases by the (a) GS, (b) ST, (c) Kreis, and (d) OF methods. The rms errors and the processing times are presented in Table $\underline{2}$. From Tables $\underline{1}$ and 2 we can see that the proposed method has accuracy similar to the rest of approaches and is approximately 1 or 2 orders of magnitude faster.

In conclusion, we have proposed a novel two-step phase-shifting method based on the GS orthonormalization method that does not need to know the phase step between interferograms. The proposed method is very fast and easy to implement. This algorithm can be especially useful in interferometry applications where the processing time is restrictive. We have tested the proposed method with simulated and real interferograms. We have compared our algorithm with the Kreis [1],

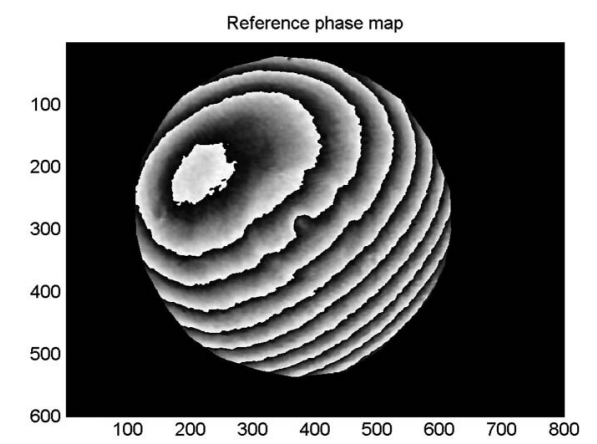

Fig. 5. Reference wrapped phase.
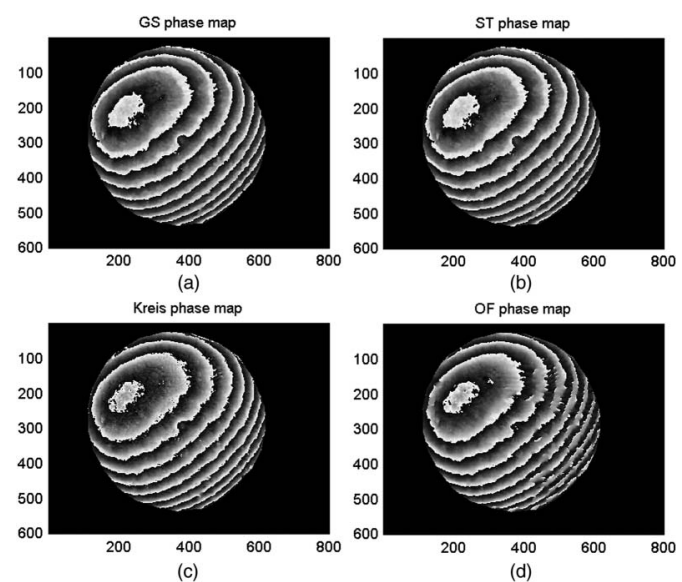

Fig. 6. Reconstructed wrapped phases by (a) the proposed GS method and the (b) ST, (c) Kreis, and (d) OF methods using real interferograms.

Table 2. Root-Mean-Square Errors and Processing Times Obtained by the GS, Kreis, ST, and OF Methods with Real Patterns

\begin{tabular}{lllll}
\hline & GS & Kreis & ST & OF \\
\hline rms (rad) & 0.38 & 0.43 & 0.38 & 0.66 \\
Time (s) & 0.069 & 0.62 & 4.0 & 1.4 \\
\hline
\end{tabular}

ST [2], and OF [3] methods and we have shown very good performance of the proposed method. All the examples of this work can be reproduced running the MATLAB package that can be found in [8]].

\section{References}

1. T. M. Kreis and W. P. O. Jueptner, Proc. SPIE 1553, 263 (1992).

2. J. Vargas, J. A. Quiroga, T. Belenguer, M. Servín, and J. C. Estrada, Opt. Express 19, 638 (2011).

3. J. Vargas, J. A. Quiroga, C. O. S. Sorzano, J. C. Estrada, and J. M. Carazo, Opt. Lett. 36, 3485 (2011).

4. J. Vargas, J. A. Quiroga, and T. Berenguer, Opt. Lett. 36, 1326 (2011).

5. J. Vargas, J. A. Quiroga, and T. Berenguer, Opt. Lett. 36, 2215 (2011).

6. G. Strang, Introduction to Linear Algebra (Wellesley Cambridge, 2009).

7. Z. Y. Wang and B. T. Han, Opt. Lett. 29, 1671 (2004).

8. http://goo.gl/IZKF3. 